\title{
High Resolution Photoexcitation Measurements Exacerbate the Long-Standing Fe XVII Oscillator Strength Problem
}

Steffen Kühn $\odot,{ }^{1,2, *}$ Chintan Shah $\odot,{ }^{3,1, \dagger}$ José R. Crespo López-Urrutia $\odot,{ }^{1}$ Keisuke Fujii $\odot,{ }^{4}$ René Steinbrügge $\odot{ }^{5}$ Jakob Stierhof $\odot,{ }^{6}$ Moto Togawa, ${ }^{1}$ Zoltán Harman, ${ }^{1}$ Natalia S. Oreshkina $\odot,{ }^{1}$ Charles Cheung $\odot,{ }^{7}$ Mikhail G. Kozlov $\odot,{ }^{8,9}$ Sergey G. Porsev $\odot,{ }^{8,7}$ Marianna S. Safronova, ${ }^{7,10}$ Julian C. Berengut $\odot,{ }^{11,1}$ Michael Rosner $\odot,{ }^{1}$ Matthias Bissinger@, ${ }^{12,6}$ Ralf Ballhausen, ${ }^{6}$ Natalie Hellø, ${ }^{13}$ SungNam Park $\odot,{ }^{14}$ Moses Chung $\odot,{ }^{14}$ Moritz Hoesch $\odot,{ }^{5}$ Jörn Seltmann, ${ }^{5}$ Andrey

S. Surzhykov $\odot,{ }^{15,16}$ Vladimir A. Yerokhin $\odot,{ }^{17}$ Jörn Wilms $\odot,{ }^{6}$ F. Scott Porter $\odot,{ }^{3}$ Thomas Stöhlker $\odot,{ }^{18,19,20}$

Christoph H. Keitel@, ${ }^{1}$ Thomas Pfeifer, ${ }^{1}$ Gregory V. Brown $\oplus^{13}{ }^{13}$ Maurice A. Leutenegger, ${ }^{3}$ and Sven Bernitt $\odot^{1,18,19,20}$

${ }^{1}$ Max-Planck-Institut für Kernphysik, Saupfercheckweg 1, 69117 Heidelberg, Germany

${ }^{2}$ Heidelberg Graduate School of Fundamental Physics, Ruprecht-Karls-Universität Heidelberg, Im Neuenheimer Feld 226, 69120 Heidelberg, Germany

${ }^{3}$ NASA/Goddard Space Flight Center, 8800 Greenbelt Rd, Greenbelt, Maryland 20771, USA

${ }^{4}$ Department of Mechanical Engineering and Science, Graduate School of Engineering, Kyoto University, Kyoto 615-8540, Japan

${ }^{5}$ Deutsches Elektronen-Sychrotron DESY, Notkestraße 85, 22607 Hamburg, Germany

${ }^{6}$ Dr. Karl Remeis-Sternwarte, Sternwartstraße 7, 96049 Bamberg, Germany

${ }^{7}$ Department of Physics and Astronomy, University of Delaware, Newark, Delaware 19716, USA

${ }^{8}$ Petersburg Nuclear Physics Institute of NRC "Kurchatov Institute", Gatchina 188300, Russia

${ }^{9}$ St. Petersburg Electrotechnical University "LETI", Prof. Popov Str. 5, St. Petersburg 197376, Russia

${ }^{10}$ Joint Quantum Institute, National Institute of Standards and Technology and the University of Maryland, Gaithersburg, Maryland 20742, USA

${ }^{11}$ School of Physics, University of New South Wales, Sydney, New South Wales 2052, Australia

${ }^{12}$ Erlangen Centre for Astroparticle Physics (ECAP), Erwin-Rommel-Straße 1, 91058 Erlangen, Germany

${ }^{13}$ Lawrence Livermore National Laboratory, 7000 East Avenue, Livermore, California 94550, USA

${ }^{14}$ Ulsan National Institute of Science and Technology, 50 UNIST-gil, Ulsan 44919, South Korea

${ }^{15}$ Physikalisch-Technische Bundesanstalt, Bundesalle 100, 38116 Braunschweig, Germany

${ }^{16}$ Braunschweig University of Technology, Universitätsplatz 2, 38106 Braunschweig, Germany

${ }^{17}$ Peter the Great St. Petersburg Polytechnic University, 195251 St. Petersburg, Russia

${ }^{18}$ Institut für Optik und Quantenelektronik, Friedrich-Schiller-Universität Jena, Max-Wien-Platz 1, 07743 Jena, Germany

${ }^{19}$ Helmholtz-Institut Jena, Fröbelstieg 3, 07743 Jena, Germany

${ }^{20}$ GSI Helmholtzzentrum für Schwerionenforschung, Planckstraße 1, 64291 Darmstadt, Germany

(Received 16 November 2019; revised manuscript received 14 February 2020; accepted 17 April 2020; published 1 June 2020)

For more than 40 years, most astrophysical observations and laboratory studies of two key soft x-ray diagnostic $2 p-3 d$ transitions, $3 C$ and $3 D$, in Fe XVII ions found oscillator strength ratios $f(3 C) / f(3 D)$ disagreeing with theory, but uncertainties had precluded definitive statements on this much studied conundrum. Here, we resonantly excite these lines using synchrotron radiation at PETRA III, and reach, at a millionfold lower photon intensities, a 10 times higher spectral resolution, and 3 times smaller uncertainty than earlier work. Our final result of $f(3 C) / f(3 D)=3.09(8)(6)$ supports many of the earlier clean astrophysical and laboratory observations, while departing by five sigmas from our own newest large-scale $a b$ initio calculations, and excluding all proposed explanations, including those invoking nonlinear effects and population transfers.

DOI: 10.1103/PhysRevLett.124.225001

Published by the American Physical Society under the terms of the Creative Commons Attribution 4.0 International license. Further distribution of this work must maintain attribution to the author(s) and the published article's title, journal citation, and DOI. Open access publication funded by the Max Planck Society.
Space x-ray observatories, such as Chandra and XMMNewton, resolve $L$-shell transitions of iron dominating the spectra of many hot astrophysical objects [1-4]. Some of the brightest lines arise from Fe XVII (Ne-like iron) around $15 \AA$ : the resonance line $3 C\left(\left[\left(2 p^{5}\right)_{1 / 2} 3 d_{3 / 2}\right]_{J=1} \rightarrow\left[2 p^{6}\right]_{J=0}\right)$ and the intercombination line $3 D \quad\left(\left[\left(2 p^{5}\right)_{3 / 2} 3 d_{5 / 2}\right]_{J=1} \rightarrow\right.$ $\left.\left[2 p^{6}\right]_{J=0}\right)$. Appearing over a broad range of plasma temperatures and densities, they are crucial for diagnostics of electron temperatures, elemental abundances, ionization 

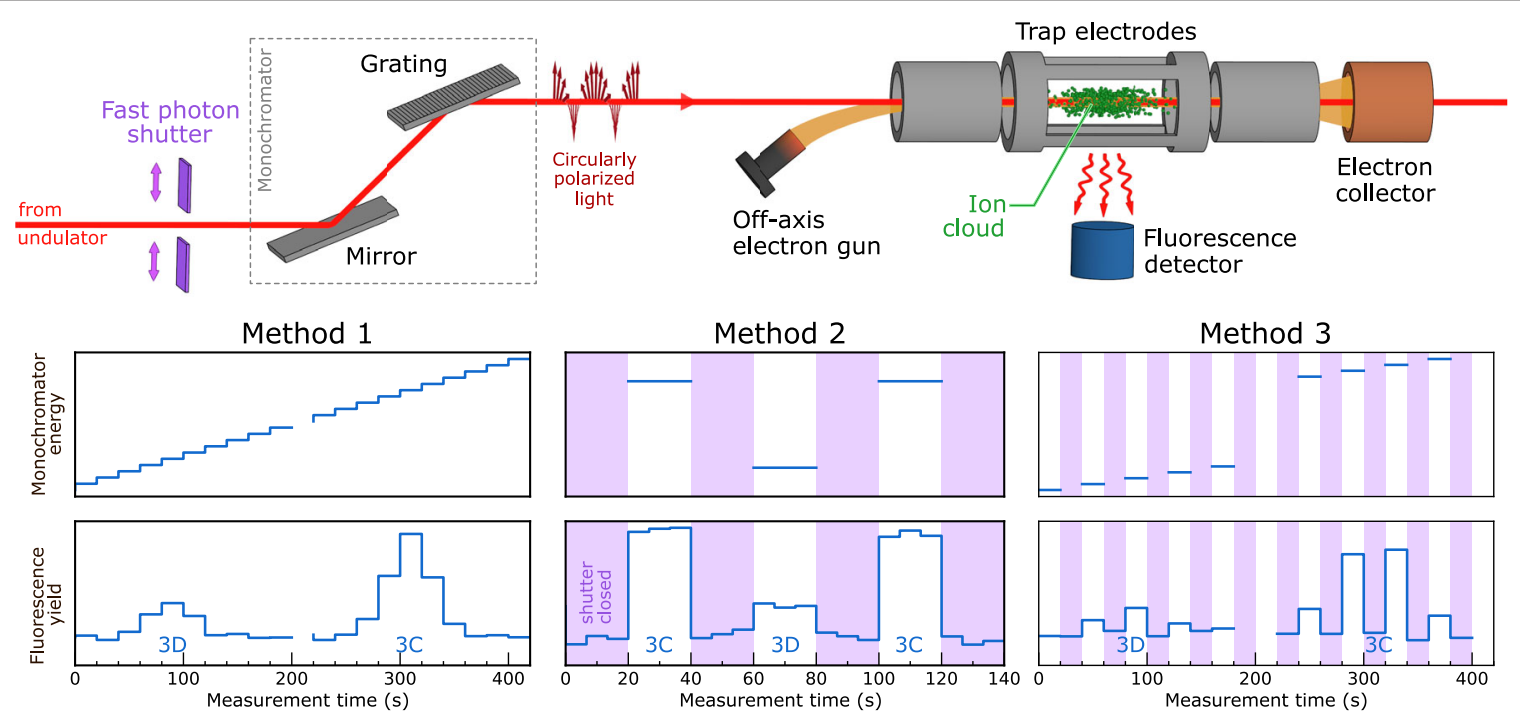

FIG. 1. (Top) Experimental setup: an electron beam (orange) aimed at the trap center produces Fe XVII ions, which are then resonantly excited by a monochromatic photon beam (red). Subsequent X-ray fluorescence is registered by a silicon drift detector. (Bottom) Fluorescence yield and photon energy vs time with three different methods: (1) line scans with open photon shutter; $(2,3)$ by closing it at each step (purple areas), we subtract the electron-beam-induced background at (2) each line center and (3) scanning over a one-fullwidth-at-half-maximum range.

conditions, velocity turbulences, and opacities [5-15]. However, for the past four decades, their observed intensity ratios persistently disagree with advanced plasma models, diminishing the utility of high resolution x-ray observations. Several experiments using electron beam ion trap (EBIT) and tokamak devices have scrutinized plausible astrophysical and plasma physics explanations as well as the underlying atomic theory [16-23], but also revealed clear departures from predictions while broadly agreeing with astrophysical observations [19,20,24]. This has fueled a long-lasting controversy on the cause being a lack of understanding of astrophysical plasmas, or inaccurate atomic data.

A direct probe of these lines using an EBIT at the Linac Coherent Light Source (LCLS) x-ray free-electron laser (XFEL) found again their oscillator strength ratio $f(3 C) /$ $f(3 D)$ to be lower than predicted, but close to astrophysical observations [25]. This highlighted difficulties with oscillator strength calculations in many-electron systems [24-31]. Nonetheless, at the high peak brilliance of the LCLS XFEL, nonlinear excitation dynamics $[32,33]$ or nonequilibrium time evolution [34] might have affected the result of Bernitt et al. [25]. An effect of resonance-induced population transfer between $\mathrm{Fe}$ XVI and $\mathrm{Fe}$ XVII ions was also postulated [35] since the $\mathrm{Fe}$ XVI line $C\left(\left[\left(2 p^{5}\right)_{1 / 2} 3 s 3 d_{5 / 2}\right]_{J=3 / 2} \rightarrow\left[2 p^{6} 3 s\right]_{J=1 / 2}\right)$ appeared blended with the Fe XVII line $3 D$. A recent semiempirical calculation [36] reproduces the LCLS results [25] by finetuning relativistic couplings and orbital relaxation effects, but its validity has been disproved [37].

In this Letter, we report on new measurements of resonantly excited Fe XVI and Fe XVII with a synchrotron source at tenfold improved spectral resolution and millionfold lower peak photon flux than in [25], suppressing nonlinear dynamical effects $[32,34,38]$ and undesired ion population transfers [35]. We also carry out improved large-scale calculations using three different advanced approaches [39-41], all showing a five-sigma departure from our experimental results.

We used the compact PolarX-EBIT [42], in which a monoenergetic electron beam emitted by an off-axis cathode (see Fig. 1) is compressed by a magnetic field. At the trap center, it collides with a beam of ironpentacarbonyl molecules, dissociating them, and producing highly charged $\mathrm{Fe}$ ions with a relative abundance of Fe XVI to Fe XVII close to unity. These ions stay radially confined by the negative space charge of the $\sim 2-\mathrm{mA}, 1610-\mathrm{eV}(\approx 3$ times the Fe XVI ionization potential) electron beam and axially by potentials applied to surrounding electrodes.

Monochromatic, circularly polarized photons from the P04 beamline [43] at the PETRA III synchrotron photon source enter through the electron gun, irradiate the trapped ions, and exit through the collector aperture. They can resonantly excite x-ray transitions on top of the strong electron-induced background due to ionization, recombination, and excitation processes. A side-on-mounted energyresolving windowless silicon drift photon detector (SDD) equipped with a 500-nm thin aluminum filter registers these emissions.

By scanning the P04 monochromator between 810 and $830 \mathrm{eV}$, we excite the Fe XVII lines $3 C$ and $3 D$, as well as the Fe XVI lines $B\left(\left[\left(2 p^{5}\right)_{1 / 2}\left(3 s 3 d_{3 / 2}\right)_{1}\right]_{J=1 / 2} \rightarrow\right.$ $\left[2 p^{6} 3 s\right]_{J=1 / 2}$ ) and $C$. They are also nonresonantly excited by electron impact as the electron beam energy is well 


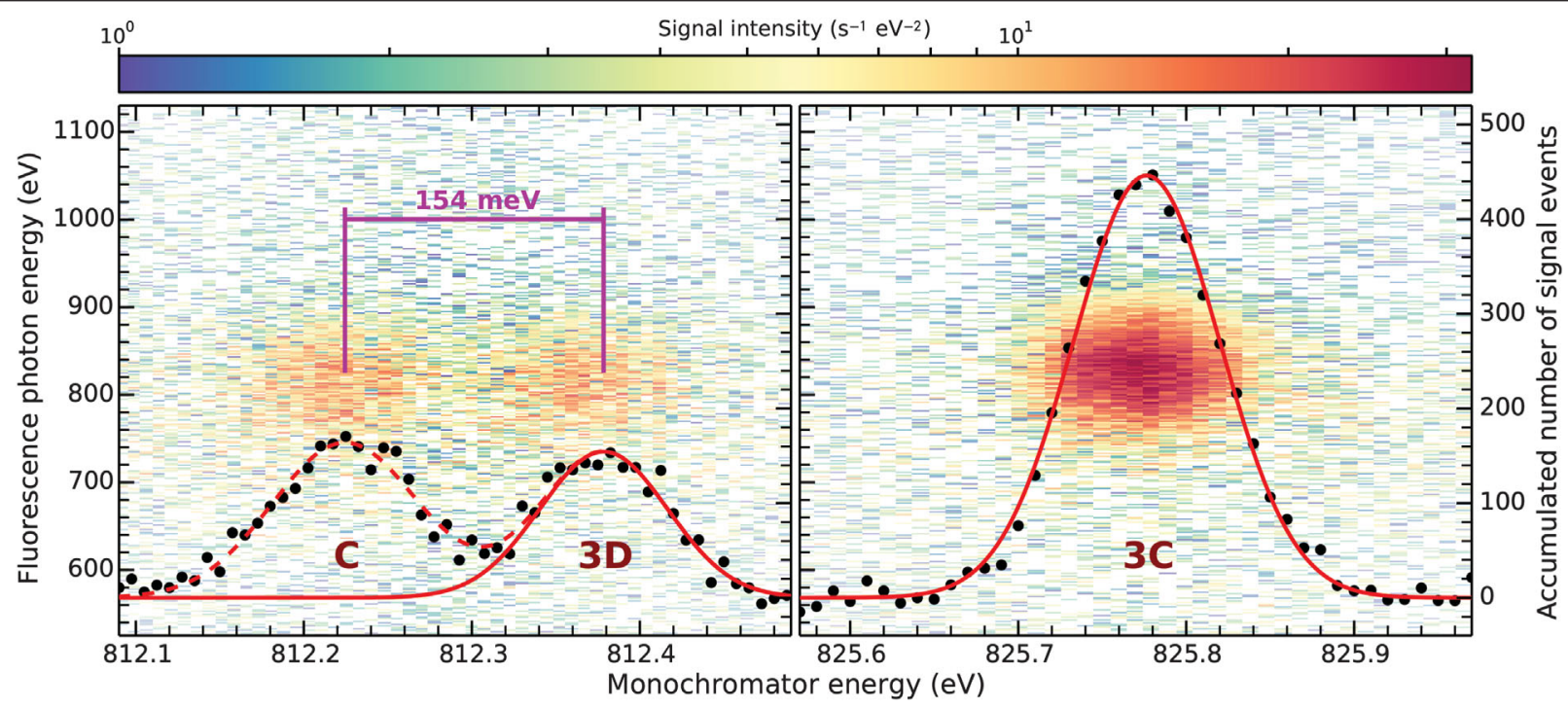

FIG. 2. Fluorescence photon yield and energy vs excitation-photon energy for the Fe XVI $C$, Fe XVII $3 C$, and $3 D$ transitions recorded by a silicon-drift detector. Black dots: total fluorescence within a $50-\mathrm{eV}$ region of interest. Red solid lines: Fits to $3 C$ and $3 D$. Red dashed line: fit to $C$.

above threshold [21,23]. This leads to a strong, nearly constant $\mathrm{x}$-ray background at the same energies as the photoexcited transitions but independent of the exciting photon-beam energy. In our earlier work [25], we rejected this background by detecting the fluorescence in time coincidence with the sub-picosecond-long LCLS pulses of $\approx 10^{11}$ photons each at $120 / \mathrm{s}$. Because of much longer and weaker 50 -ps-long pulses $\left(\approx 10^{3}\right.$ photons, $6 \times 10^{6} / \mathrm{s}$ repetition rate) at $\mathrm{P} 04$ and the limited time resolution of SDD, we could not use the coincidence method and reached a signal-to-background ratio of only $\sim 5 \%$. To improve this, we used a shutter to cyclically turn on and off the P04 photon beam.

Using a 50- $\mu \mathrm{m}$ slit width, we reached a resolving power of $E / \Delta E \approx 10000,10-15$ times higher than that of Chandra and XMM-Newton grating spectrometers [44,45], and tenfold that of our previous experiment [25] (see the Supplemental Material [46]). We find a separation of $3 C$ from $3 D$ of $\Delta E_{3 C-3 D}=13.398(1) \mathrm{eV}$ and resolve for the first time the Fe XVII $3 D$ line from the Fe XVI $C$ one at $\Delta E_{3 D-C}=154.3(1.3) \mathrm{meV}$. This gives us the $3 C / 3 D$ intensity ratio without having to infer a contribution of Fe XVI line $C$ (in [25] still unresolved) from the intensity of the well-resolved Fe XVI A line. Thereby, we largely reduce systematic uncertainties and exclude the resonanceinduced population transfer mechanism [35] that may have affected the LCLS result [25].

We apply three different methods (Fig. 1) to systematically measure the $3 C / 3 \mathrm{D}$ oscillator strength ratio. In method 1, we did not operate the photon shutter; instead, we repeatedly scanned the lines $C$ and $3 D(812.0-$ $812.5 \mathrm{eV})$, as well as $3 C(825.5-826.0 \mathrm{eV})$, in both cases using scans of 100 steps with 20-s exposure each (see
Fig. 2). The SDD fluorescence signal was integrated over a $50-\mathrm{eV}$ wide photon-energy region of interest (ROI) comprising $3 C, 3 D$, and $C$ and recorded while scanning the incident photon energy. By fitting Gaussians to the scan result, we obtain line positions, widths, and yields, modeling the electron-impact background as a smooth linear function [23]. The ratio of $3 C$ and $3 D$ areas is then proportional to the oscillator strength ratio [33]. However, given the low 5\% signal-to-background ratio and long measurement times, changes in the background cause systematic uncertainties. In method 2, we fixed the monochromator energy to the respective centroids of $C$, $3 D$, and $3 C$ found with method 1 and cyclically opened and closed the shutter for equal periods of $20 \mathrm{~s}$ to determine the background. The background-corrected fluorescence yields at the line peaks were multiplied with the respective linewidths from method 1 , to obtain the $3 C / 3 D$ ratio. Still, slow monochromator shifts from the selected positions could affect the results. To address this, in method 3, we scanned across the FWHM of $C, 3 D$, and $3 C$ in 33 steps with on-off exposures of $20 \mathrm{~s}$, which reduced the effect of possible monochromator shifts. After background subtraction, we fit Gaussians to the lines of interest fixing their widths to values from method 1 .

All three methods share systematic uncertainties caused by energy-dependent filter transmission and detector efficiency $(\sim 1 \%)$ and by the incident photon beam flux variation $(\sim 2 \%)$. Additionally, for method 1 , we estimate systematic uncertainties from background $(\sim 1.2 \%)$ and ROI selection $(\sim 2.7 \%)$. In method 2, possible monochromator shifts from (set) line centroids and widths taken from method 1 cause a systematic uncertainty of $\sim 3.5 \%$. Analogously, for method 3, we estimate a 3\% uncertainty 


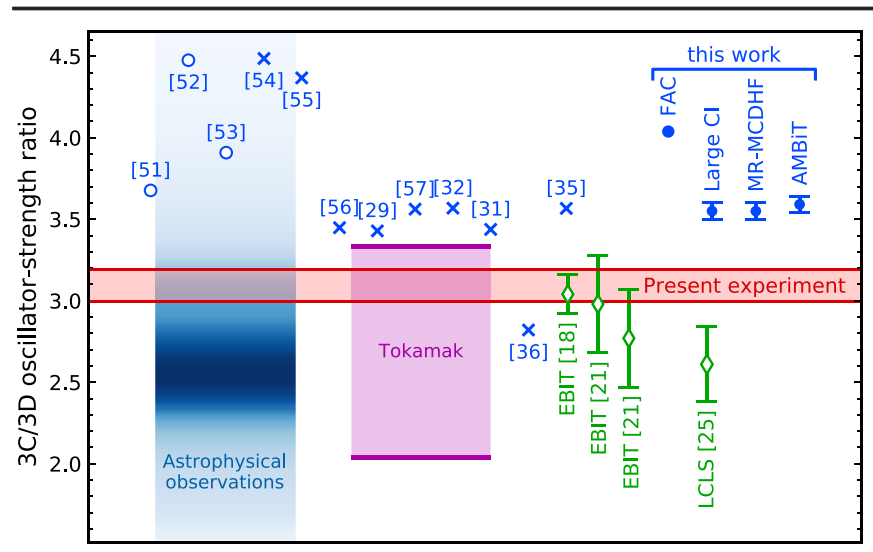

FIG. 3. Present experimental $3 C / 3 D$ ratios compared with previous predictions and experiments. Red band: combined results of the three different methods. Blue open circles: values from databases [51-53]. Blue crosses: predictions [29,31, $32,35,36,54-57]$. Note that the validity of theory [36] has been disputed [37]. Blue solid circles: present FAC [58], large-scale CI [39], MR-MCDHF [40], and AMBiT [41] calculations. Blue band: observed line ratios in astrophysical sources [3,4,7,59-61], with color shades coding the distribution of values weighted by their reported accuracies. Purple band: spread of tokamak results [20]. Open green diamonds: previous EBIT results [18,21,25]. Note that the spread seen in various astrophysical sources and in tokamak in part arises from insufficient removal of Fe XVI $C$ line contamination of Fe XVII $3 D$ line, at varying Fe XVI/Fe XVII abundance ratios [6,20]; nonlinear dynamical effects [32,34,38] (see main text) may have reduced the LCLS ratio [25].

due to the use of linewidth constraints from method 1 . The weighted average of all three methods is $f(3 C) /$ $f(3 D)=3.09(8)_{\text {sys }}(6)_{\text {stat }}$; see Fig. 3 (see the Supplemental Material [46] for individual ratio and uncertainties). Note that the circular polarization of the photon beam does not affect these results, since $3 C$ and $3 D$ (both $\Delta J=1$ ) share the same angular emission characteristics [47-50].

Calculations using a density-matrix approach by Oreshkina et al. [32,33], and the time-dependent collisional-radiative model of Loch et al. [34], pointed to a possible nonlinear response of the excited upper state populations in [25], reducing the observed oscillator strength ratio [38], which would depend on photon pulse parameters, like intensity, duration, and spectral distribution. It has been estimated that peak intensities above $10^{12} \mathrm{~W} / \mathrm{cm}^{2}$ would give rise to nonlinear effects $[32,38]$. Fluctuations of the self-amplified spontaneous emission process at LCLS can conceivably generate some pulses above that threshold. At P04, we estimate a peak intensity of $\approx 10^{5} \mathrm{~W} / \mathrm{cm}^{2}$, more than 6 orders of magnitude below that threshold (see the Supplemental Material [46]). This could explain why the $3 C / 3 D$ ratio in Bernitt et al. [25] is in slight disagreement with the present result. Nonetheless, our experiment validates the main conclusion of that work with reduced uncertainty. Our work implies that future experiments at ultrabrilliant light sources should take possible nonlinear effects into account.

In the present work, we also carried out relativistic calculations using a very-large-scale configuration interaction (CI) method, correlating all ten electrons, including Breit and quantum electrodynamical [62] corrections. We implemented a message passing interface (MPI) version of the CI code from [39] to increase the number of configurations to over 230000 , saturating the computation in all possible numerical parameters. Basis sets of increasing size are used to check for convergence, with all orbitals up to $12 s p 17 \mathrm{dfg}$ included in the largest version (the contributions of $n>12 s p$ orbitals are negligible). We start with all possible single and double excitations from the $2 s^{2} 2 p^{6}$, $2 s^{2} 2 p^{5} 3 p$ even and $2 s^{2} 2 p^{5} 3 s, 2 s^{2} 2 p^{5} 3 d, 2 s 2 p^{6} 3 p$, $2 s^{2} 2 p^{5} 4 d, 2 s^{2} 2 p^{5} 5 d$ odd configurations, correlating eight electrons. We separately calculate triple excitations and fully correlate the $1 s^{2}$ shell, and also included dominant quadruple ones, finding them negligible. The line strengths $S$ and $3 C / 3 D$ oscillator strength ratio after several computation stages are summarized in the Supplemental Material [46] to illustrate the small effect of all corrections. Theoretical uncertainties are estimated based on the variance of results from the smallest to largest runs, size of the various effects, and small variances in the basis set construction. We verified that the energies of all 18 states considered, counted from the ground state, agree with the National Institute for Standards and Technology [51] database well within the experimental uncertainty of $0.05 \%$. The theoretical $3 C-3 D$ energy difference of $13.44 \mathrm{eV}$ is in agreement with the experiment to $0.3 \%$.

We also carried out entirely independent large-scale calculations using the multireference multiconfiguration Dirac-Hartree-Fock (MR-MCDHF) approach [40] with up to 1.2 million configurations. First, the $2 s^{2} 2 p^{5} 3 s, 2 s^{2} 2 p^{5} 3 d$, and $2 s 2 p^{6} 3 p J=1$ levels were used as reference states to generate the list of configuration state functions with single and double exchanges from all occupied orbitals up to $12 s p d f g h i$. Virtual orbitals were added in a layer-by-layer manner. Subsequently, the role of triple excitations was studied by the CI method. In a second step, the multireference list was extended to include all $J=1$ odd parity states, generated from the Ne-like ground state by single and double electron exchanges. Monitoring the convergence of the results for the addition of layers of virtual orbits, we arrive at an oscillator strength ratio of 3.55(5) and to a $3 C-3 D$ energy splitting of $13.44(5) \mathrm{eV}$. Another full-scale CI calculation with more than a million configurations was carried out in the particle-hole formalism using AMBiT [41], agreeing well with the other theoretical results. Full details of all calculations can be found in the Supplemental Material [46].

We emphasize that there are no other known quantum mechanical effects or numerical uncertainties to consider within the CI and MCDHF approaches. With modern 
computational facilities and MPI codes, we have shown that all other contributions are negligible at the level of the quoted theoretical uncertainties. The significant improvements in experimental and theoretical precision reported here have only further deepened this long-standing problem. This work on the possibly so far most intensively studied many-electron ion in experiment and theory, finally demonstrates convergence of the dedicated atomic calculations on all possible parameters, excluding an incomplete inclusion of the correlation effects as potential explanation of this puzzle.

Our result is the presently most accurate on the $3 C / 3 D$ oscillator strength ratio. Its excellent resolution suggests promising direct determinations of the natural linewidth. They depend on the Einstein $A$ coefficients, hence, on the oscillator strengths [63]. Thus, future accurate measurements of individual natural linewidths of $3 C$ and $3 D$ not only would test theory more stringently than their oscillator strength ratio does, but also deliver accurate oscillator strengths.

Moreover, $3 C$ and $3 D$ with their, among many transitions, strong absorption and emission rates can also dominate the Planck and Rosseland mean opacity of hot plasmas [64-66]. Therefore, an accurate determination of their oscillator strengths may help elucidating the iron opacity issue [12,14,67], if, e.g., Rosseland mean opacity models $[68,69]$ were found to use predicted oscillator strengths also in departure from experiments. Our result exposes in simplest dipole-allowed transitions of Fe XVII a far greater issue, namely, the persistent problems in the best approximations in use, and calls for renewed efforts in further developing the theory of many-electron systems.

Shortcomings of low-precision atomic theory for $L$-shell ions had already emerged in the analysis of high resolution Chandra and XMM-Newton data $[13,19,20,24,70]$. Similar inconsistencies were recently found in the high resolution $K$-shell x-ray spectra of the Perseus cluster recorded with the Hitomi microcalorimeter [71,72]. Moreover, recent opacity measurements $[12,14]$ have highlighted serious inconsistencies in the opacity models used to describe the interiors of stars, which have to rely on calculated oscillator strengths.

All this shows that the actual accuracy and reliability of the opacity and turbulence velocity diagnostics are still uncertain, and with them, the modeling of hot astrophysical and high-energy density plasmas. The upcoming x-ray observatory missions XRISM [73] and Athena [74] will require improved and quantitatively validated modeling tools for maximizing their scientific harvest. Thus, benchmarking atomic theory in the laboratory is vital. As for the long-standing Fe XVII oscillator strength problem, our results may be immediately used to semiempirically correct spectral models of astrophysical observations.

Financial support was provided by the Max-PlanckGesellschaft and Bundesministerium für Bildung und Forschung through Project No. 05K13SJ2. Work by
C.S. was supported by the Deutsche Forschungsgemeinschaft Project No. 266229290 and by an appointment to the NASA Postdoctoral Program at the NASA Goddard Space Flight Center, administered by Universities Space Research Association under contract with NASA. Work by LLNL was performed under the auspices of the U.S. Department of Energy under Contract No. DE-AC5207NA27344 and supported by NASA grants. M. A. L. and F. S. P. acknowledge support from NASA's Astrophysics Program. The work of M. G. K. and S. G. P. was supported by the Russian Science Foundation under Grant No. 19-1200157. The theoretical research was supported in part through the use of Information Technologies resources at the University of Delaware, specifically the high-performance Caviness computing cluster. The work of C. C. and M. S. S. was supported by USA NSF Grant No. PHY1620687. Work by U.N.I.S. T. was supported by the National Research Foundation of Korea (Grant No. NRF-2016R1A5A1013277). J. C. B. acknowledges support from the Alexander von Humboldt Foundation. We acknowledge DESY (Hamburg, Germany), a member of the Helmholtz Association HGF, for the provision of experimental facilities. Parts of this research were carried out at PETRA III.

S. K. and C. S. contributed equally to this work.

*steffen.kuehn@mpi-hd.mpg.de †chintan.d.shah@nasa.gov

[1] F. B. S. Paerels and S. M. Kahn, Annu. Rev. Astron. Astrophys. 41, 291 (2003).

[2] C. R. Canizares, D. P. Huenemoerder, D. S. Davis, D. Dewey, K. A. Flanagan, J. Houck, T. H. Markert, H. L. Marshall, M. L. Schattenburg, N. S. Schulz et al., Astrophys. J. Lett. 539, L41 (2000).

[3] E. Behar, J. Cottam, and S. M. Kahn, Astrophys. J. 548, 966 (2001).

[4] R. Mewe, A. J. J. Raassen, J. J. Drake, J. S. Kaastra, R. L. J. van der Meer, and D. Porquet, Astron. Astrophys. 368, 888 (2001).

[5] J. H. Parkinson, Astron. Astrophys. 24, 215 (1973).

[6] R. Doron and E. Behar, Astrophys. J. 574, 518 (2002).

[7] H. Xu, S. M. Kahn, J. R. Peterson, E. Behar, F. B. S. Paerels, R. F. Mushotzky, J. G. Jernigan, A. C. Brinkman, and K. Makishima, Astrophys. J. 579, 600 (2002).

[8] N. S. Brickhouse and J. Schmelz, Astrophys. J. Lett. 636, L53 (2006).

[9] J. S. Sanders and A. C. Fabian, Mon. Not. R. Astron. Soc. 412, L35 (2011).

[10] T. Kallman, A. E. Daniel, H. Marshall, C. Canizares, A. Longinotti, M. Nowak, and N. Schulz, Astrophys. J. 780, 121 (2014).

[11] J. de Plaa, I. Zhuravleva, N. Werner, J. S. Kaastra, E. Churazov, R. K. Smith, A. J. J. Raassen, and Y. G. Grange, Astron. Astrophys. 539, A34 (2012). 
[12] J. E. Bailey, T. Nagayama, G. P. Loisel, G. A. Rochau, C. Blancard, J. Colgan, P. Cosse, G. Faussurier, C. Fontes, F. Gilleron et al., Nature (London) 517, 56 (2015).

[13] P. Beiersdorfer, N. Hell, and J. Lepson, Astrophys. J. 864, 24 (2018).

[14] T. Nagayama, J. E. Bailey, G. P. Loisel, G. S. Dunham, G. A. Rochau, C. Blancard, J. Colgan, P. Cossé, G. Faussurier, C. J. Fontes et al., Phys. Rev. Lett. 122, 235001 (2019).

[15] L. Gu, A. J. J. Raassen, J. Mao, J. de Plaa, C. Shah, C. Pinto, N. Werner, A. Simionescu, F. Mernier, and J. S. Kaastra, Astron. Astrophys. 627, A51 (2019).

[16] G. V. Brown, P. Beiersdorfer, D. A. Liedahl, K. Widmann, and S. M. Kahn, Astrophys. J. 502, 1015 (1998).

[17] P. Beiersdorfer, S. von Goeler, M. Bitter, and D. B. Thorn, Phys. Rev. A 64, 032705 (2001).

[18] G. V. Brown, P. Beiersdorfer, H. Chen, M. H. Chen, and K. J. Reed, Astrophys. J. Lett. 557, L75 (2001).

[19] P. Beiersdorfer, E. Behar, K. Boyce, G. Brown, H. Chen, K. Gendreau, M.-F. Gu, J. Gygax, S. Kahn, R. Kelley et al., Astrophys. J. Lett. 576, L169 (2002).

[20] P. Beiersdorfer, M. Bitter, S. Von Goeler, and K. Hill, Astrophys. J. 610, 616 (2004).

[21] G. V. Brown, P. Beiersdorfer, H. Chen, J. H. Scofield, K. R. Boyce, R. L. Kelley, C. A. Kilbourne, F. S. Porter, M. F. Gu, S. M. Kahn, and A. E. Szymkowiak, Phys. Rev. Lett. 96, 253201 (2006).

[22] G. V. Brown and P. Beiersdorfer, Phys. Rev. Lett. 108, 139302 (2012).

[23] C. Shah, J. R. C. López-Urrutia, M. F. Gu, T. Pfeifer, J. Marques, F. Grilo, J. P. Santos, and P. Amaro, Astrophys. J. 881, 100 (2019).

[24] M. F. Gu, arXiv:0905.0519.

[25] S. Bernitt, G. V. Brown, J. K. Rudolph, R. Steinbrugge, A. Graf, M. Leutenegger, S. W. Epp, S. Eberle, K. Kubicek, V. Mackel et al., Nature (London) 492, 225 (2012).

[26] U. I. Safronova, C. Namba, I. Murakami, W. R. Johnson, and M. S. Safronova, Phys. Rev. A 64, 012507 (2001).

[27] G. X. Chen and A. K. Pradhan, Phys. Rev. Lett. 89, 013202 (2002).

[28] S. D. Loch, M. S. Pindzola, C. P. Ballance, and D. C. Griffin, J. Phys. B 39, 85 (2006).

[29] G.-X. Chen, Phys. Rev. A 76, 062708 (2007).

[30] G. Chen, Phys. Rev. A 84, 012705 (2011).

[31] J. A. Santana, J. K. Lepson, E. Träbert, and P. Beiersdorfer, Phys. Rev. A 91, 012502 (2015).

[32] N. S. Oreshkina, S. M. Cavaletto, C. H. Keitel, and Z. Harman, Phys. Rev. Lett. 113, 143001 (2014).

[33] N. S. Oreshkina, S. M. Cavaletto, C. H. Keitel, and Z. Harman, J. Phys. B 49, 094003 (2016).

[34] S. D. Loch, C. P. Ballance, Y. Li, M. Fogle, and C. J. Fontes, Astrophys. J. Lett. 801, L13 (2015).

[35] C. Wu and X. Gao, Sci. Rep. 9, 7463 (2019).

[36] C. Mendoza and M. A. Bautista, Phys. Rev. Lett. 118, 163002 (2017).

[37] K. Wang, P. Jönsson, J. Ekman, T. Brage, C. Y. Chen, C. F. Fischer, G. Gaigalas, and M. Godefroid, Phys. Rev. Lett. 119, 189301 (2017).
[38] Y. Li, M. Fogle, S. Loch, C. Ballance, and C. Fontes, Can. J. Phys. 95, 869 (2017).

[39] M. Kozlov, S. Porsev, M. Safronova, and I. Tupitsyn, Comput. Phys. Commun. 195, 199 (2015).

[40] C. F. Fischer, G. Gaigalas, P. Jönsson, and J. Biero, Comput. Phys. Commun. 237, 184 (2019).

[41] E. V. Kahl and J. C. Berengut, Comput. Phys. Commun. 238, 232 (2019).

[42] P. Micke, S. Kühn, L. Buchauer, J. R. Harries, T. M. Bücking, K. Blaum, A. Cieluch, A. Egl, D. Hollain, S. Kraemer et al., Rev. Sci. Instrum. 89, 063109 (2018).

[43] J. Viefhaus, F. Scholz, S. Deinert, L. Glaser, M. Ilchen, J. Seltmann, P. Walter, and F. Siewert, Nucl. Instrum. Methods Phys. Res., Sect. A 710, 151 (2013).

[44] J. Den Herder, A. Brinkman, S. Kahn, G. BranduardiRaymont, K. Thomsen, H. Aarts, M. Audard, J. Bixler, A. den Boggende, J. Cottam et al., Astron. Astrophys. 365, L7 (2001).

[45] C. R. Canizares, J. E. Davis, D. Dewey, K. A. Flanagan, E. B. Galton, D. P. Huenemoerder, K. Ishibashi, T. H. Markert, H. L. Marshall, M. McGuirk et al., Publ. Astron. Soc. Pac. 117, 1144 (2005).

[46] See Supplemental Material at http://link.aps.org/ supplemental/10.1103/PhysRevLett.124.225001 for details.

[47] V. V. Balashov, A. N. Grum-Grzhimailo, and N. M. Kabachnik, Polarization and Correlation Phenomena in Atomic Collision (Kluwer Academics/Plenum Publishers, Dordrecht, 2000).

[48] J. K. Rudolph, S. Bernitt, S. W. Epp, R. Steinbrügge, C. Beilmann, G. V. Brown, S. Eberle, A. Graf, Z. Harman, N. Hell et al., Phys. Rev. Lett. 111, 103002 (2013).

[49] C. Shah, H. Jörg, S. Bernitt, S. Dobrodey, R. Steinbrügge, C. Beilmann, P. Amaro, Z. Hu, S. Weber, S. Fritzsche et al., Phys. Rev. A 92, 042702 (2015).

[50] C. Shah, P. Amaro, R. Steinbrügge, S. Bernitt, J. R. C. López-Urrutia, and S. Tashenov, Astrophys. J. Suppl. Ser. 234, 27 (2018).

[51] A. Kramida, Yu. Ralchenko, J. Reader, and NIST ASD Team, NIST atomic spectra database (version 5.6.1). https:// physics.nist.gov/asd (2019). National Institute of Standards and Technology, Gaithersburg, MD, 2018.

[52] J. S. Kaastra, R. Mewe, and H. Nieuwenhuijzen, in 11th Colloq. on UV and X-ray Spectroscopy of Astrophysical and Laboratory Plasmas, edited by K. Yamashita and $\mathrm{T}$. Watanabe (Universal Academy Press, Tokyo, 1996), pp. 411-414.

[53] A. R. Foster, L. Ji, R. K. Smith, and N. S. Brickhouse, Astrophys. J. 756, 128 (2012).

[54] A. Bhatia and G. Doschek, At. Data Nucl. Data Tables 52, 1 (1992).

[55] G.-X. Chen, A. K. Pradhan, and W. Eissner, J. Phys. B 36, 453 (2003).

[56] C. Z. Dong, L. Y. Xie, S. Fritzsche, and T. Kato, Nucl. Instrum. Methods Phys. Res., Sect. B 205, 87 (2003).

[57] P. Jönsson, P. Bengtsson, J. Ekman, S. Gustafsson, L. Karlsson, G. Gaigalas, C. F. Fischer, D. Kato, I. Murakami, H. Sakaue et al., At. Data Nucl. Data Tables 100, 1 (2014).

[58] M. F. Gu, Can. J. Phys. 86, 675 (2008). 
[59] R. Blake, T. Chubb, H. Friedman, and A. Unzicker, Astrophys. J. 142, 1 (1965).

[60] D. L. McKenzie, P. B. Landecker, R. M. Broussard, H. R. Rugge, R. M. Young, U. Feldman, and G. A. Doschek, Astrophys. J. 241, 409 (1980).

[61] J.-U. Ness, J. H. M. M. Schmitt, M. Audard, M. Güdel, and R. Mewe, Astron. Astrophys. 407, 347 (2003).

[62] I. I. Tupitsyn, M. G. Kozlov, M. S. Safronova, V. M. Shabaev, and V. A. Dzuba, Phys. Rev. Lett. 117, 253001 (2016).

[63] V. Weisskopf and E. P. Wigner, Berechnung der natürlichen Linienbreite auf Grund der Diracschen Lichttheorie, in Part I: Particles and Fields. Part II: Foundations of Quantum Mechanics, edited by A.S. Wightman (Springer Berlin Heidelberg, Berlin, Heidelberg, 1997), pp. 30-49.

[64] F. J. Rogers and C. A. Iglesias, Science 263, 50 (1994).

[65] M. J. Seaton, Y. Yan, D. Mihalas, and A. K. Pradhan, Mon. Not. R. Astron. Soc. 266, 805 (1994).

[66] P. Beiersdorfer, A. L. Osterheld, V. Decaux, and K. Widmann, Phys. Rev. Lett. 77, 5353 (1996).
[67] J. E. Bailey, G. A. Rochau, C. A. Iglesias, J. Abdallah, J. J. MacFarlane, I. Golovkin, P. Wang, R. C. Mancini, P. W. Lake, T. C. Moore et al., Phys. Rev. Lett. 99, 265002 (2007).

[68] C. Fontes, C. Fryer, A. Hungerford, P. Hakel, J. Colgan, D. Kilcrease, and M. Sherrill, High Energy Density Phys. 16, 53 (2015).

[69] J.-C. Pain, F. Gilleron, and M. Comet, Atoms 5 (2017).

[70] H. Netzer, Astrophys. J. 604, 551 (2004).

[71] Hitomi Collaboration et al., Nature (London) 535, 117 (2016).

[72] Hitomi Collaboration et al., Publ. Astron. Soc. Jpn. 70, 12 (2018).

[73] M. Tashiro, H. Maejima, K. Toda, R. Kelley, L. Reichenthal, J. Lobell, R. Petre, M. Guainazzi, E. Costantini, M. Edison et al., Proc. SPIE Int. Soc. Opt. Eng. 10699, 1069922 (2018).

[74] D. Barret, T. L. Trong, J.-W. Den Herder, L. Piro, X. Barcons, J. Huovelin, R. Kelley, J. M. Mas-Hesse, K. Mitsuda, S. Paltani et al., Proc. SPIE 10699, 106991G (2018). 